\section{Neoplastic Fever in a Cancer Patient: A Case Report and Review of Literature}

\author{
Arshad Hussain Shah*, Muzaffar UI Sultan Mattoo and Mustfa M \\ Al Mubarak
}

Department of Hemato-Oncology, King Saud Medical City Riyadh, Saudi Arabia

\begin{abstract}
Fever is common symptom presented in cancerous patient, either through state of infection or developed by tumour itself in certain malignancies. At many instances it is described as para neoplastic syndrome in patient with lymphoma, acute leukemias and renal cell carcinoma.Neoplastic fever is characterised as intermittent fever, with no signs of rigors, chills and any mental disturbance. The alternate fever spikes and regular pulse rate is monitored for confirmatory diagnosis. Certain diagnostic imaging are necessary for definite diagnosis. CT scan and MRI are essential for tumour location and identification. Adjuvant diagnostic aid are naproxen test, ESR and blood hormones test. Treatment modalities comprises of surgical therapy, radiotherapy and chemotherapy. A case of a squamous cell lung cancer patient who was initially evaluated and managed as a case of pyrexia of unknown origin.
\end{abstract}

Keywords: Neoplastic fever; Para-neoplastic syndrome; Pyrexia of unknown origin; Squamous cell lung cancer

\section{Introduction}

Fever is an evident sign of inflammatory reactions taking place in the body. It is a well known common symptom observed in $67 \%$ of the infectious cases in contrast, neoplastic fever arises from the tumour itself or invasive procedure [1]. Neoplastic fever is a paraneoplastic syndrome that originates from cancer. In $27 \%$ of cases, the fever is associated with non-infectious febrile episodes [1]. Neoplastic fever is a unique feature in certain malignancies such as hematological

*Corresponding author: Arshad Hussain Shah, Department of Hemato-Oncology, King Saud Medical City Riyadh, Saudi Arabia, Tel: +966 592540013; E-mail: drarshadoncologiest@gmail.com; a.rasmy@ksmc.med.sa

Citation: Shah AH, Mattoo MUIS, Mubarak MMAI (2020) Neoplastic Fever in a Cancer Patient: A Case Report and Review of Literature. J Clin Stud Med Case Rep 7: 085.

Received: March 16, 2020; Accepted: March 24, 2020; Published: March 31, 2020

Copyright: (c) 2020 Shah AH, et al. This is an open-access article distributed under the terms of the Creative Commons Attribution License, which permits unrestricted use, distribution, and reproduction in any medium, provided the original author and source are credited. malignancies, colon cancer, renal cell carcinoma, and cholangiocarcinoma [2]. However, an unusual case of non-small cell lung carcinoma was presented in the outpatient department with a high-grade intermittent fever at the stage of diagnosis that was later suggested as neoplastic fever.

Fever is divided into two categories septicemic infection in a cancer patient or non-infectious condition arising from the tumour itself. The neoplastic fever accounts for $20 \%$ of patients with an undetermined origin. It is important to diagnose the disease at the initial stage and provides a cure on time for minimal morbidity and mortality. During the management of cancer, it becomes critical for the doctors to present a definite diagnosis as the fever could be a side effect of chemotherapy, cancer or the underlying infection. At the time of growth and development of the tumour the body is vulnerable to infection; therefore, fever as a result of the infection is associated with the tumor in each case. Neoplastic fever is characterised as an intermittent fever with alternative febrile periods over a period. In non-infectious condition, a visible change noticed is the alterations of the oral mucosa, mucositis mainly occurring due to medication, blood transfusions, radiation, endocrine disturbances, surgery, and neoplastic fever [3]. It is recommended by the surgeons to surgically remove the mass in addition to follow-ups with radiotherapy and chemotherapy to avoid future relapse. The neoplastic fever disappears within $24 \mathrm{hrs}$ after the surgical treatment assuring definite diagnosis of neoplastic fever.

\section{Case Report}

A 44-year-old Filipino married women reported to our outpatient department with History of recurrent high grade fever, weight loss and occasional dry cough. The patient was admitted and investigated thoroughly. She underwent series of investigations including blood, urine and sputum cultures. She was also screened for tuberculosis, fungal and viral infections. But all these investigations couldn't detect any kind of infective factor. During all this period she was put on empirical antibiotics for 2 weeks with a broader coverage, and antifungal for 7 days. But with no significant clinical improvement and no relief in fever, while the high-grade fever continued to persist. Naproxen was also tried and had limited effect of short-term relief. Her initial X Ray chest had a small suspicious small right upper lobe lesion, which was reported as consolidation path. But it couldn't disappear with antibiotics and antifungal treatment. So, a CT Scan and bronchoscopy was planned, which revealed a right upper lobe mass $4 \mathrm{~cm} \times 3 \mathrm{~cm}$ on right upper lobe with associated mediastinal lymphadenopathy. To confirm tissue diagnosis, an image guided biopsy was performed that confirmed the malignancy as squamous cell carcinoma. The fever was between 38 degrees to 40 degrees without associated chills and rigors. Her blood test showed high Erythrocyte Sedimentation Rate (ESR) of $80 \mathrm{~mm} / \mathrm{h}$ and the White Blood Cell Count (WBC) was 24109/1.

Patient was planned for chemotherapy based on taxeme's and platinum. After first cycle of chemotherapy, the fever vanished for 6 days. It was first time since more than one month that the fever subsided 
for several days, it recurred again but with decreased frequency and intensity. Also Blood tests show some improvement with decrease in WBC, ESR levels. This type of fever is commonly termed as neoplastic fevers. It is a type of paraneoplastic syndrome. So, her paraneoplastic syndrome (neoplastic fever) responded to chemotherapy with relief in fever after one month of persistent fever.

\section{Discussion}

The neoplastic fever is a unique type of fever that can only be treated by the removal of the cause, cancer. The pathophysiology of the fever manifestation is still unknown. As per Fever Of Unknown Origin (FUO) classification if any patient has fever more than 38.3 for more than 3 weeks without a definite known cause is obliged to undergo certain sets of investigation [4,5]. This type of fever accounts for $27 \%$ of non-infectious illness [5].

Authors have reported that patient with cancer commonly shows sign of intermittent fever [2,5]. In addition, there are other causes also for fever which include drug or transfusion reaction, central nervous system metastasis, and chemotherapy or radiation-induced fever [4]. Zhang, et al. [3], 67\% of patient account for infectious fever in cancer patients while $27 \%$ neoplastic fever. It is suggested that early diagnosis is a key step for fever palliation, lowering morbidity and refraining from the unnecessary expenditure for detailed diagnosis and treatment [6]. Intermittent fever is not a diagnostic indication for neoplastic fever. It is compulsory to monitor the pulse rate regularly. Comparing with bacteraemia, the bacteria produce toxins, which stimulate the immune system causing the cytokines fluctuation. neoplastic cancer showed an increase in pulse rate during febrile period in contrast to bacterial infection.

In majority of patient there is no fever pattern, which is suggestive of pathognomic of cancer. Clinical manifestation for this fever is either during the day or night accompanied by night sweats [6]. Consistent presence of the fever indicates neoplastic disease. Any signs of sudden fever with rigors and chills indicates bacteraemia. Any fever noted without concomitant rise in the pulse is known as Faget's sign that indicates drug fever, use of beta-blocker drugs, or infection with intracellular organisms. Similarly, fever observed in the afternoon is suggestive of an intraabdominal abscess [7].

Cancer patients prolonging hospitalisation period often cause infections, which might be the leading reason for the induced fever [8]. Moreover, blood transfusions and medicinal administration equally causes similar symptoms. The explanation for such induced fever is the immunological reactions in the body to the antigens or the bacteria. In the case of viral and bacterial infections, the acute and convalescent serologies including the urine test that has $70 \%$ sensitivity for detecting the infections $[3,9]$. In contrast for diagnosing the neoplastic fever, acute phase reactants supplemental testing offers reliable source for definite diagnosis. Many laboratory tests conducted are nonspecific in nature. The acute phase reactants testing includes the ESR, a systematic nonspecific test that measures the length a drop of blood falls within a microtube in one hour [10]. The results greater than $100 \mathrm{~mm} / \mathrm{hr}$ confirms the diagnosis of the tumour, autoimmune infection or hidden infection [10]. ESR offers a general overview of the systematic inflammation; however, does not provide definite diagnosis. In addition, similar results can be observed evaluating the levels of C-reactive protein [11].
Metastasis is commonly associated with the primary tumour masses such as lymphadenopathy. Lymph node examination presented with tender, fixed enlarged lymph nodes indicated reactive hyperplasia. The increase in the infiltrative cells was due to the induction from the neoplastic cells. A further explanation for neoplastic cancer could be the tumour necrosis, dead tissue releasing the pyrogens or the TNF release [12]. Our patient test result showed no significant evidence of necrosis of tumour. In certain cases, the brain metastasis invades the brain tissue leading to activation of phospholipase A2 causing the neurogenic fever [13]. However, no such metastasis was suspected.

The pathophysiology is still a question regarding neoplastic fever. One of the explanations for neoplastic fever is that either the tumour cells or the host cells are intrigued by the tumour to produce some pyrogenic cells such as Interleukin (IL)-1, IL-6, IL-8 Tumor Necrosis Factor (TNF)- $\alpha$, and interferon [11]. These cytokines induce a change in the hypothalamus to raise the set point temperature through alteration in prostaglandin E2 levels [9]. The detailed histopathological examination of our patient revealed the dedifferentiated carcinoma infiltrated by a large number of lymphocytes and multiple aggregated PMNs.

Confirmatory diagnosis can be achieved through radiological imaging. The latest diagnostic modality used to detect the abnormal masses is through computed topographical scanning. Neoplastic patients who presented with fever revealed primary tumour masses, metastatic lesion and body cavity effusions, pneumonia, hydronephrosis, biliary dilatation, and lymphdadenopathy $[2,13]$. Best way to use these modalities is in combination with needle biopsy in cancer patients. Mostly recommended in febrile patients suspected of the underlying tumour. Furthermore, MRI, magnetic resonance imaging is recommended in the condition where there is soft tissue neoplasm in muscle, bone sarcomas and brain tumour. In our patient only CT scan was sufficient to diagnose the lesion in the lung.

The administration of antibiotics is a way to discriminate between neoplastic fever and infectious fever. Infection fever is characterised as rigors, temperature, and periodic sweating occasionally accompanied by tachycardia, hypotension, and mental status changes, particularly in gram-negative bacteraemia. however, neoplastic fever is usually described as a feeling of warmth and night sweats but less frequently by chills, tachycardia, and mental status changes [14]. Furthermore, pertinent to laboratory tests, it displays that the CRP and ESR levels do not provide evident differentiation between the two types of fever. Authors have reported that procalcitonin levels are raised in case of bacteraemia than cancerous tumour [1,7]. Alsirafy, et al. tried differentiating the two types of fever by closely monitoring the effect of anti-inflammatory, naproxen to reduce the fever within $24 \mathrm{hrs}$. the positive response was achieved [12]; however, some demonstrated conflicting results.

Definitive diagnosis is important to be achieved to proceed with appropriate treatment modalities such as surgical operation, radiotherapy, and chemotherapy. Studies have portrayed that $41 \%$ of the patients responded to systematic anti-neoplastic therapy [3]. NSAIDS have equally displayed superior efficacy in treating neoplastic fever. Our patient showed a timely reduction in the fever; however, it reoccurred when the NSAIDS were withdrawn. Kudlowitz, et al. [14], explained that as the tumour increases in the size it increases the production of IL 6 . Thus, the naproxen does not show promising effect 
in reducing the size of the tumour; however, it can cause haematological disturbance as a side effect of NSAID [14]. Therefore, the use of naproxen should be used in a controlled form other randomised trial compared indomethacin and diclophenac sodium that displayed comparable results in contrast naproxen has a quick effect [15].

These types of fevers are distressful for the patients causing delirium and fatigue. Intense clinical investigation and diagnostic tests necessary builds a great deal of stress. In addition, the chemotherapy in the patient with extensive metastasis causes some adverse effects. Neutropenic fever is a common complication of chemotherapy. Surgeons have administered certain growth factors such as Granulocyte Colony Stimulating Factor (G-CSF) and Granulocyte-Macrophage Colony-Stimulating Factor (GM-CSF) to control the neutropenic fever [8]. Research in the US has successfully shown that filgrastim was able to control the episodes of neutropenic fever $[3,8]$. Likewise, studies in Europe also displayed similar results. In contrast, the steroid component has a palliative effect. The NSAIDS have shown symptomatic cure. The use of naproxen at a certain point is considered a distinguishing feature between neoplastic fever and non-neoplastic fever. In some patients, naproxen provides fever lysis. The previous study has shown 7 out of 10 patients demonstrated lysis of the fever after the withdrawal within 24hrs [12]. The dose of both chemotherapy and naproxen can be reduced once the patients start responding to the therapy.

The evidence of naproxen effects is limited due to small sample sizes in the study. Before the administration of naproxen, it is essential to perform physical and laboratory and diagnostic test including empiric antibiotic treatment and imaging. This often leads to prolonging hospitalisation and extensive evaluation in the case of advanced cancer. The dose is a critical factor for treatment in any condition. A dose of $250 \mathrm{mg}$ twice a day displayed a success rate of 98.1\% [12]. Moreover, small dose of $125 \mathrm{mg}$ naproxen, $375 \mathrm{mg}$ twice a day, and $250 \mathrm{mg} 3$ times a day is considered useful. Doctors have recommended 125 to $750 \mathrm{mg} /$ day, stabilising the concentration level after 3 days [3]. if the fever persists than naproxen should be discontinued and further evaluation will be required for the treatment.

Studies have displayed that $60 \%$ of the patient did not present with fever until after their admission to the hospital. Few patients exhibited unique symptoms as those enlisted for neoplastic fever such as high peak fever, chills, and leucocytosis. Physicians have always recommended to administer antibiotic, measure the vital signs, and blood and other cultures monitoring necessary to exclude possible fatal infections. A study demonstrated that $92 \%$ of the patient showed one spike daily in fever commonly observed between the time 9 am to 5 $\mathrm{pm}$ [9]. $28 \%$ of patients showed a spike twice a day between 9 am and $9 \mathrm{pm}$ and 9 am and $5 \mathrm{pm}[9]$.

Literature states that circadian rhythm is maintained throughout the body in health and febrile period $[9,15]$. Statistical analysis displayed high levels during the night and low levels during early morning [9]. In addition, cortisol levels were measured in certain trials presenting with high peaks in the morning while lower in the night. Therefore, circadian rhythm of cytokines was successfully proven to be related to neoplasm. In case of gastro intestinal cancer cortisol and TNF-a displayed a significant relation with circadian rhythm fluctuation [2,11]. In the advanced cancer patient a self-regulation system between neuroendocrine system, the hypothalamic-pituitary adrenal axis, and the cytokine network activates. Further investigation proved that cortisol rhythm had a positive relation with elevated levels of serum cytokines in colorectal cancer. Limper, et al. reported that cancer and its treatment causes a release of proinflammatory cytokines [7], flat levels of cortisol, reduced sensitivity of glucocorticoid, lower cortisol response to stress, and activates the cytokine signal pathway such as Nuclear Factor-Kappa B (NFkB) and p38 mitogen activated protein kinase in the brain. Any fluctuation in the cytokine levels and reduction in the cortisol sensitivity has shown to have a profound influence in the neoplastic fever pattern.

Tumor growth, cancer treatment, and related complications cause a rise in the expression of inflammatory cytokines and NF-kB activation. However, the fluctuating pulse rate is not addressed as a confirmatory value unless monitored over a period. Chemotherapy instigates the production of cytokines. 7 percent of the patients in a study showed transient elevated pulse rates after discontinuation of post chemotherapy dexamethasone although the cessation does not reduce the cytokines level [9]. Under such circumstances, fever origin from infection or neoplasia is critical to differentiate. Naproxen and corticosteroids displayed antipyretic effect on neoplastic fever. bearing in mind NFkb has a profound effect on the number of cytokine production. The use of NSAIDS caused the suppression of NFkb and COX thus palliating the fever. In contrast, naproxen was found in $87 \%$ of patient to show complete lysis of neoplastic fever [9]. The febrile stage response to the corticosteroids by blocking the activity of phospholipase A2 leading of reduction in PGE2 synthesis and production of pyrogenic cytokines can be stopped through blocking the transcription of the mRNA. In 14\% of patient's dexamethasone mask the neoplastic fever during cancer therapy [9]. An unusual behaviour was noted in the patient who did not respond to naproxen demonstrated lysis of fever after chemotherapy. Therefore, it was stated that a septic fever do not respond to naproxen. Nevertheless, $10-20 \%$ of the patient failed to respond to complete lysis of fever after naproxen administration [3,9]. However, NSAID exhibited successful lysis of neoplastic fever in $38 \%$ patient with no neoplasm.

\section{Conclusion}

Our patient displayed symptoms that were indicative of neoplastic fever; however, all necessary steps were followed as suggested by physicians for definitive diagnosis. This unusual case points out the chances of a unique and rare condition to exhibit a well-known symptom.

\section{Reference}

1. Shi N, Xing C, Chang X, Dai M, Zhao Y (2016) Pancreatic carcinoma masked as fever of unknown origin: A case report and comprehensive review of literature. Medicine (Baltimore) 95: 4643.

2. Pasikhova Y, Ludlow S, Baluch A (2017) Fever in patients with cancer. Cancer Control 24: 193-197.

3. Zhang H, Wu Y, Lin Z, Zhong X, Liu T, et al. (2019) Naproxen for the treatment of neoplastic fever: A PRISMA-compliant systematic review and meta-analysis. Medicine (Baltimore) 98: 15840.

4. Unger M, Karanikas G, Kerschbaumer A, Winkler S, Aletaha D (2016) Fever of unknown origin (FUO) revised. Wiener Klinische Wochenschrift 128: 796-801.

5. Loizidou A, Aoun M, Klastersky J (2016) Fever of unknown origin in cancer patients. Crit Rev Oncol Hematol 101: 125-30. 
6. Yaegashi H, Izumi K, Kitagawa Y, Kadono Y, Konaka H, et al. (2014) Differential diagnosis between bacterial infection and neoplastic fever in patients with advanced urological cancer: the role of procalcitonin. Int J Urol 21:104-106.

7. Limper M, De Kruif M, Duits A, Brandjes D, van Gorp E (2010) The diagnostic role of procalcitonin and other biomarkers in discriminating infectious from non-infectious fever. J Infect 60: 409-416.

8. van der Velden WJ, Herbers AH, Netea MG, Blijlevens NM (2014) Mucosal barrier injury, fever and infection in neutropenic patients with cancer: introducing the paradigm febrile mucositis. Br J Haematol 167: 441-452.

9. Liaw CC, Huang JS, Chen JS, Chang JW,Chang HK, et al. (2010) Using vital sign flow sheets can help to identify neoplastic fever and other possible causes in oncology patients: a retrospective observational study. J Pain Symptom Manage 40: 256-65.

10. Markanday A (2015) Acute phase reactants in infections: evidence-based review and a guide for clinicians. Open Forum Infect Dis 2: 98.
11. Nakamura T, Matsumine A, Matsubara T, Asanuma K, Sudo A (2016) Neoplastic fever in patients with bone and soft tissue sarcoma. Mol Clin Oncol 5: 631-634.

12. Alsirafy SA, El Mesidy SM, Abou-Elela EN, Elfaramawy YI (2011) Naproxen test for neoplastic fever may reduce suffering. J Palliat Med 14: 665-667.

13. Zee Y, Soo R (2010) Non-small cell lung cancer presenting with neoplastic fever at diagnosis and relapse. International Journal of Infectious Diseases 14: 518-521.

14. Kudlowitz D (2014) Neoplastic Fever: Pathophysiology, Clinical Features, And Diagnostic Assessment. The NYU Langone Online Journal of Medicine.

15. Zasowska-Nowak A, Ciałkowska-Rysz A (2017) Neoplastic fever. Medycyna Paliatywna Vol: 9. 


\section{di \\ нетан}

Advances In Industrial Biotechnology | ISSN: 2639-5665

Advances In Microbiology Research | ISSN: 2689-694X

Archives Of Surgery And Surgical Education | ISSN: 2689-3126

Archives Of Urology

Archives Of Zoological Studies | ISSN: 2640-7779

Current Trends Medical And Biological Engineering

International Journal Of Case Reports And Therapeutic Studies | ISSN: 2689-310X Journal Of Addiction \& Addictive Disorders | ISSN: 2578-7276

Journal Of Agronomy \& Agricultural Science | ISSN: 2689-8292

Journal Of AIDS Clinical Research \& STDs | ISSN: 2572-7370

Journal Of Alcoholism Drug Abuse \& Substance Dependence | ISSN: 2572-9594

Journal Of Allergy Disorders \& Therapy | ISSN: 2470-749X

Journal Of Alternative Complementary \& Integrative Medicine | ISSN: 2470-7562

Journal Of Alzheimers \& Neurodegenerative Diseases | ISSN: 2572-9608

Journal Of Anesthesia \& Clinical Care | ISSN: 2378-8879

Journal Of Angiology \& Vascular Surgery | ISSN: 2572-7397

Journal Of Animal Research \& Veterinary Science | ISSN: 2639-3751

Journal Of Aquaculture \& Fisheries | ISSN: 2576-5523

Journal Of Atmospheric \& Earth Sciences | ISSN: 2689-8780

Journal Of Biotech Research \& Biochemistry

Journal Of Brain \& Neuroscience Research

Journal Of Cancer Biology \& Treatment | ISSN: 2470-7546

Journal Of Cardiology Study \& Research | ISSN: 2640-768X

Journal Of Cell Biology \& Cell Metabolism | ISSN: 2381-1943

Journal Of Clinical Dermatology \& Therapy | ISSN: 2378-8771

Journal Of Clinical Immunology \& Immunotherapy | ISSN: 2378-8844

Journal Of Clinical Studies \& Medical Case Reports | ISSN: 2378-8801

Journal Of Community Medicine \& Public Health Care | ISSN: 2381-1978

Journal Of Cytology \& Tissue Biology | ISSN: 2378-9107

Journal Of Dairy Research \& Technology | ISSN: 2688-9315

Journal Of Dentistry Oral Health \& Cosmesis | ISSN: 2473-6783

Journal Of Diabetes \& Metabolic Disorders | ISSN: 2381-201X

Journal Of Emergency Medicine Trauma \& Surgical Care | ISSN: 2378-8798

Journal Of Environmental Science Current Research | ISSN: 2643-5020

Journal Of Food Science \& Nutrition | ISSN: 2470-1076

Journal Of Forensic Legal \& Investigative Sciences | ISSN: 2473-733X

Journal Of Gastroenterology \& Hepatology Research | ISSN: 2574-2566
Journal Of Genetics \& Genomic Sciences | ISSN: 2574-2485

Journal Of Gerontology \& Geriatric Medicine | ISSN: 2381-8662

Journal Of Hematology Blood Transfusion \& Disorders | ISSN: 2572-2999

Journal Of Hospice \& Palliative Medical Care

Journal Of Human Endocrinology | ISSN: 2572-9640

Journal Of Infectious \& Non Infectious Diseases | ISSN: 2381-8654

Journal Of Internal Medicine \& Primary Healthcare | ISSN: 2574-2493

Journal Of Light \& Laser Current Trends

Journal Of Medicine Study \& Research | ISSN: 2639-5657

Journal Of Modern Chemical Sciences

Journal Of Nanotechnology Nanomedicine \& Nanobiotechnology | ISSN: 2381-2044

Journal Of Neonatology \& Clinical Pediatrics | ISSN: 2378-878X

Journal Of Nephrology \& Renal Therapy | ISSN: 2473-7313

Journal Of Non Invasive Vascular Investigation | ISSN: 2572-7400

Journal Of Nuclear Medicine Radiology \& Radiation Therapy | ISSN: 2572-7419

Journal Of Obesity \& Weight Loss | ISSN: 2473-7372

Journal Of Ophthalmology \& Clinical Research | ISSN: 2378-8887

Journal Of Orthopedic Research \& Physiotherapy | ISSN: 2381-2052

Journal Of Otolaryngology Head \& Neck Surgery | ISSN: 2573-010X

Journal Of Pathology Clinical \& Medical Research

Journal Of Pharmacology Pharmaceutics \& Pharmacovigilance | ISSN: 2639-5649

Journal Of Physical Medicine Rehabilitation \& Disabilities | ISSN: 2381-8670

Journal Of Plant Science Current Research | ISSN: 2639-3743

Journal Of Practical \& Professional Nursing | ISSN: 2639-5681

Journal Of Protein Research \& Bioinformatics

Journal Of Psychiatry Depression \& Anxiety | ISSN: 2573-0150

Journal Of Pulmonary Medicine \& Respiratory Research | ISSN: 2573-0177

Journal Of Reproductive Medicine Gynaecology \& Obstetrics | ISSN: 2574-2574

Journal Of Stem Cells Research Development \& Therapy | ISSN: 2381-2060

Journal Of Surgery Current Trends \& Innovations | ISSN: 2578-7284

Journal Of Toxicology Current Research | ISSN: 2639-3735

Journal Of Translational Science And Research

Journal Of Vaccines Research \& Vaccination | ISSN: 2573-0193

Journal Of Virology \& Antivirals

Sports Medicine And Injury Care Journal | ISSN: 2689-8829

Trends In Anatomy \& Physiology | ISSN: 2640-7752

Submit Your Manuscript: https://www.heraldopenaccess.us/submit-manuscript 